\title{
Isolation And Antibiotic Susceptibility Pattern With Esbl Production Of Klebsiella Pneumoniae Isolated From Sputum, Pus And Urine Samples In A Tertiary Care Hospital.
}

\author{
Dr D. Vijaya Bharathi*, Dr Supriya Panda**, Dr K Bhaskara Rao** \\ *Assistant Professor, ** Professor, \\ Dept. Of Microbiology, Maharajah's Institute Of Medical Sciences, Nellimarla, Vizianagaram, Ap, India
}

\begin{abstract}
Introduction: Klebsiella pneumoniae has been associated with different types of infections and one of the most important aspects of Klebsiella is the emergence of multi drug resistant strains particularly those involved in nosocomial diseases.

Materials and methods: A total number of 800 sputum, 148 pus and 850 urine samples from September 2013 to August 2014 were included in the study. Isolates of Klebsiella pneumoniae were identified by their standard microbiological techniques and were subjected to antibiotic sensitivity testing by modified Kirby-Bauer disc diffusion method.

Results: Of the 1798 samples processed 410 (22\%) samples were culture positive for various

organisms. A total of 410 organisms were isolated, of them 157 (38\%) were Klebsiella pneumoniae. The frequency of ESBL producers in our study was 70 (44\%) of all Klebsiella isolates.

Conclusion: The present study reveals $8.73 \%$ of isolation rate of Klebsiella pneumoniae from various clinical samples with highest sensitivity to amikacin and highest resistance to cefotaxime and ceftriaxone. Prevalence of ESBLs among members of Enterobacteriaceae constitutes a serious threat to the current beta-lactam therapy, leading to treatment failure. There is an urgent need to emphasize rational use of drugs to minimize the misuse of available antimicrobials.
\end{abstract}

\section{Introduction}

Klebsiella pneumoniae (Klebsiella) are ubiquitously present and reported worldwide. In recent years, Klebsiella have become important pathogens in nosocomial infections ${ }^{[1]}$, which have been well documented in United States ${ }^{[2]}$ and India ${ }^{[3]}$. Epidemic and endemic nosocomial infections caused by Klebsiella species are leading causes of morbidity and mortality ${ }^{[4]}$

Klebsiella pneumoniae is a Gram-negative, non-motile, encapsulated, lactose fermenting, facultative anaerobic, rod shaped bacterium found in the normal flora of the mouth, skin and intestines ${ }^{[5]}$ Klebsiella is most frequently recovered from clinical specimens and can cause a classic form of primary pneumonia and a variety of extrapulmonary infections, including enteritis and meningitis in infants, urinary tract infections in children and adults and septicaemia ${ }^{[6]}$.

Extensive use of broad-spectrum antibiotics in hospitalized patients has led to both increased carriage of Klebsiella and the development of multidrug-resistant strains that produce extended-spectrum betalactamases (ESBLs) ${ }^{[7]}$. In recent years, there has been an increase in the incidence and prevalence of ESBLs ${ }^{[8]}$.

ESBLs have emerged as a major problem in hospitalized patients worldwide and have been involved in epidemic outbreaks in many institutions in Europe and USA and constitute a serious threat to the current $\beta$ lactam therapy as these enzymes cause resistance to most penicillins, cephalosporins and aztreonam ${ }^{[9]}$. ESBLs are distinguished into more than 30 types based on their physical properties and all are inhibited by clavulanate, sulbactam and tazobactam, a property which has been used to detect them in the laboratory ${ }^{[10]}$.

Reports of treatment failure of nosocomial infections due to ESBL-producing organism are emerging. The ESBL-producing organisms are often multidrug resistant, as the plasmids producing ESBLs can carry resistance to other antibiotics ${ }^{[11]}$

The objective of this study was to assess the prevalence of Klebsiella from different clinical specimens, their antibiotic susceptibility pattern and the extended spectrum beta-lactamase (ESBL) production among such isolates.

\section{Materials and Methods}

This study was carried from September 2013 to August 2014 at the Department of Microbiology in Maharajah's Institute of Medical Sciences, Vizianagaram. A total of 800 sputum, 148 pus and 850 urine samples were collected during this period with universal safety precautions and were transported to the laboratory 
without delay. The pus samples were either aspirated by disposable syringes or collected onto sterile cotton tipped swabs. Sputum and clean voided midstream urine was collected into screw top containers ${ }^{[12]}$.

Sputum, pus and urine samples were inoculated on Blood agar and Mac Conkey agar and incubated overnight at $37^{\circ} \mathrm{C}$. Klebsiella pneumoniae strains were identified by their morphology and biochemical characteristics. Morphology of Klebsiella pneumoniae identified were large, dome-shaped, mucoid colonies on blood agar and lactose fermenting colonies on Mac Conkey agar. In Gram-staining, Gram-negative, short, plump, straight rods were seen. The biochemical characters identified were negative indole test, negative methyl red test, positive Voges-Proskauer test, positive citrate utilization test, positive urease test, acid and abundant gas production from glucose, lactose, sucrose, maltose and mannitol sugar fermentation tests.$^{[5]}$

Antimicrobial susceptibility testing: Antibiotic sensitivity of clinical Klebsiella pneumoniae isolates was done by Bauer's and Kirby's disc diffusion method according to the CLSI guidelines 2015 (CLSI, 2015) ${ }^{[13]}$. Commercially available antibiotics, amoxyclav - $10 \mathrm{mcg}$, amikacin - 30mcg, ampicillin+sulbactum $10 \mathrm{mcg} / 10 \mathrm{mcg}$, cefotaxime - $30 \mathrm{mcg}$, ceftriaxone - 30mcg, gentamicin - $10 \mathrm{mcg}$, ofloxacin - $5 \mathrm{mcg}$ were used.

\section{NCCLS screening test}

Isolates showing an inhibition zone size of $\leq 25 \mathrm{~mm}$ with cefriaxone $(30 \mu \mathrm{g})$ and $\leq 27 \mathrm{~mm}$ with cefotaxime (30 $\mu \mathrm{g})$ were identified as potential ESBL producers. (NCCLS Screening test $)^{[14]}$.

\section{Results and Discussion}

A total of 1798 sputum, pus and urine samples were processed during our study period. Four hundred ten bacterial isolates were obtained from culture positive samples (Table-1). Of them 157 (38\%) were Klebsiella pneumoniae (Table -2). The sensitivity and resistance pattern of the Klebsiella isolates to various antibiotics in our study is shown in the Table-3.

Of the 157 Klebsiella isolated, 70 (44 \%) were ESBL producers, 13 isolates were from pus samples, constituting 52\% of all pus isolates, 37 were from sputum samples constituting $39 \%$ of all sputum isolates and 20 were from urine samples constituting $52 \%$ of all urine samples(Table- 4 ).

Risk factors associated with ESBL production include - prolonged hospital or intensive care stay, use of multiple courses of antimicrobial therapy, particularly extended spectrum cephalosporins. Hence, indiscriminate use of cephalosporins and broad spectrum antibiotics should be avoided.

In the present study culture positivity for Klebsiella in sputum was $50.2 \%$ which is little higher than Asati et al $(2013)^{[15]}$ followed by $31.14 \%$ in urine samples which is in agreement with R.Sarath Babu et al $(2012)^{[16]}$ and $24.75 \%$ in pus samples, which is similar to Valarmathi et al $(2013)^{[17]}$. Most of the isolates in our study were not multidrug resistant. The frequency of ESBL producers in our study was 70 (44\%) of all Klebsiella isolates.

Our study showed good sensitivity to amikacin, accounting for $89 \%$ of sputum and $88 \%$ of pus samples. Sensitivity to ampicillin and sulbactam was good in urine samples accounting to $85 \%$. Sensitivity to quinolones was moderate in our study.

\section{Conclusion}

The present study reveals $8.73 \%$ of isolation rate of Klebsiella pneumoniae from various clinical samples with highest sensitivity to amikacin and highest resistance to cefotaxime and ceftriaxone. ESBL isolates of Enterobacteriaceae family is a major problem worldwide. Indiscriminate use of third generation cephalosporins to treat Gram negative bacterial infection is partly responsible for the emergence of resistance to beta - lactam antibiotics. Strict adherence to the hospital antibiotic policy and good infection control practices can play a significant role in reducing the emerging drug resistance. However only screening tests were performed for detection of ESBLs in our study, confirmatory studies are required for further evaluation.

\section{References}

[1]. P. Nordamann, G. Cuzon and T. Naas "The real threat of Klebsiella pneumonia carbapenemase-producing bacteria", Lancet Infec Dis.,2009,9 (4):228-236.

[2]. J.R.Graybill, L.W. Marshall, P. Charache, C.K. Wallace and V.K.Melwin "Nosocomial pneumonia: A continuing major problem", ,Am.Rev.Respir. Dis., 1973, 108:1130-1140.

[3]. N.B. Mathur, A. Khalib , R. Sarkar and R.K. Puri " Mortality in neonatal septicaaemia with involvement of mother in management”, Ind. J. Pediatri, 1991, 28 (ii) 1259-1264.

[4]. S.J. Cryz, R. Furer and R. Germanier "Protection against fatal Klebsiella pneumonia burn wound sepsis by passive transfer of anticapsular polysaccharide", Infect. Immun., 1985, 45: 139-142.

[5]. Elmer W. Koneman, Stephen D. Allen, Willian M. janda, Paul C.Schreckenberger and Washington C.Winn. 2006. Color Atlas And Textbook Of Diagnostic Microbiology.6th edition. Baltimore : Lippincott Williams Wilkins.

[6]. Arti Kapil. 2013. Ananthanarayan \& Paniker s Textbook of Microbiology. 9th edition. Chennai: Universities Press Pvt Ltd. 
Isolation And Antibiotic Susceptibility Pattern With ESBL Production Of Klebsiella Pneumoniae ..

[7]. Dean AD, Dean AJ, Burton AH, Dicker RC; Epi-Infoversion 5: a word processing database and statistics programme for epidemiology on microcomputers.VSD.Inc Stone Mountain Ga, 1990.

[8]. Rodrigues C, Joshi P, Jani SH, Alphonse M, Radhakrishnan R, Mehta A. Detection of beta lactamase in nosocomial gram-negative clinical isolates. Indian J Med Microbiol 2004;22:247-50.

[9]. Thomson KS, Sanders CC. Detection of extended-sprectrum $\beta$-lactamases in members of the family Enterobacteriaceae. Antimicrob Agents Chemother 1992;36:1877-82.

[10]. Livermore D. Extended Spectrum -Lactamases: Clinical threat or academic curiosity? Beta-watch, Issue-7. (Meditech Media Ltd., Langley Court, London), 1993.

[11]. Livermore DM. beta-lactamases in laboratory and clinical resistance. Clin Microbiol Rev 1995; 8:557-84.

[12]. Patricia M. Tille. 2014. Bailey \& Scott s DiagnosticMicrobiology.13 $3^{\text {th }}$ edition.St.Louis,Missouri : Mosby Elsevier publications.

[13]. CLSI 2015. The Clinical and Laboratory Standards Institute (CLSI): Performance Standard for Antimicrobial Susceptibility Testing. $25^{\text {th }}$ Informational supplement. CLSI document. M100-S25.

[14]. National Committee for Clinical Laboratory Standards. Performance standards for antimicrobial disk susceptibility test, 7th edition. Approved standards, NCCLS Document M2- A7, Vol; 20 no 1; Wayne PA. 2000.

[15]. Asati Rakesh Kumar.2013. Antimicrobial Sensitivity Pattern of Klebsiella Pneumoniae isolated from Sputum from Tertiary Care Hospital, Surendranagar, Gujarat and Issues Related to the Rational Selection of Antimicrobials .Sch. J. App. Med. Sci., 2013; 1(6):928 -933

[16]. R Sarath Babu, T.V.Ramani, K.Bhaskara Rao and Supriya Panda. 2012. Antibiotic susceptibility pattern of Klebsiella pneumoniae isolated from sputum, urine and pus samples. IOSR Journal of Pharmacy and Biological Sciences (IOSRJPBS). 1(2): 04-09.

[17]. Valarmathi, S., M. Rajasekara Pandian and B. Senthilkumar. 2013. Incidence and screening of wound infection causing microorganisms. J. Acad. Indus. Res. 1(8): 508.

Table.1: Total number of organisms isolated

\begin{tabular}{|l|l|l|l|}
\hline Sample & $\begin{array}{l}\text { Total number } \\
\text { of samples }\end{array}$ & $\begin{array}{l}\text { Number of culture } \\
\text { positives }\end{array}$ & of culture positives \\
\hline Sputum & 800 & 187 & $23.3 \%$ \\
\hline Pus & 148 & 101 & $68.2 \%$ \\
\hline Urine & 850 & 122 & $22 \%$ \\
\hline Total & 1798 & 410 & \\
\hline
\end{tabular}

Table 2: Culture positivity of Klebsiella pneumoniae

\begin{tabular}{|l|l|l|l|}
\hline Sample & Total number of isolates & Klebsiella positivity & Percentage \\
\hline Sputum & 187 & 94 & $50.2 \%$ \\
\hline Pus & 101 & 25 & $24.75 \%$ \\
\hline Urine & 122 & 38 & $31.14 \%$ \\
\hline Total & 410 & 157 & $38 \%$ \\
& & & \\
\hline
\end{tabular}

Table 3: Antibiotic sensitivity pattern of Klebsiella pneumoniae

\begin{tabular}{|l|l|l|l|l|l|l|}
\hline \multirow{2}{*}{ Antibiotic } & \multicolumn{2}{l|}{ Sputum } & \multicolumn{2}{l|}{ Pus } & \multicolumn{2}{l|}{ Urine } \\
\cline { 2 - 7 } & $\mathrm{R} \%$ & $\mathrm{~S} \%$ & $\mathrm{R} \%$ & $\mathrm{~S} \%$ & $\mathrm{R} \%$ & $\mathrm{~S} \%$ \\
\hline Ceftriaxone & $76 \%$ & $24 \%$ & $64 \%$ & $36 \%$ & $68 \%$ & $32 \%$ \\
\hline Cefotaxime & $88 \%$ & $12 \%$ & $64 \%$ & $36 \%$ & $63 \%$ & $37 \%$ \\
\hline Amoxyclav & $79 \%$ & $21 \%$ & $52 \%$ & $48 \%$ & $57 \%$ & $43 \%$ \\
\hline Ampicillin Sulbactam & $34 \%$ & $66 \%$ & $12 \%$ & $78 \%$ & $15 \%$ & $85 \%$ \\
\hline Gentamicin & $36 \%$ & $64 \%$ & $32 \%$ & $68 \%$ & $52 \%$ & $48 \%$ \\
\hline Amikacin & $11 \%$ & $89 \%$ & $12 \%$ & $88 \%$ & $36 \%$ & $64 \%$ \\
\hline Ofloxacin & $25 \%$ & $75 \%$ & $20 \%$ & $80 \%$ & $63 \%$ & $37 \%$ \\
\hline
\end{tabular}


Isolation And Antibiotic Susceptibility Pattern With ESBL Production Of Klebsiella Pneumoniae ..

Table 4: ESBL positivity of Klebsiella isolates

\begin{tabular}{|l|l|l|l|}
\hline Sample & Klebsiella isolates & ESBLpositivity & $\%$ of ESBLPositivity \\
\hline Sputum & 94 & 37 & $39 \%$ \\
\hline Pus & 25 & 13 & $52 \%$ \\
\hline Urine & 38 & 20 & $52 \%$ \\
\hline Total & 157 & 70 & $44 \%$ \\
\hline
\end{tabular}

